CHAPTER XIV

ABSORPTION IN QUASI-STELLAR OBJECTS 


\title{
QSO ABSORPTION LINES AND COSMOLOGY
}

\author{
Wallace L. W. Sargent \\ Palomar Observatory, California Institute of Technology \\ Pasadena, Ca. 91125 U.S.A.
}

\begin{abstract}
The properties of the different types of QSO absorption systems are briefly summarized. An overview is given of the potential applications of absorption lines in cosmology. Recent work on the cosmological evolution of the different types of absorbers is discussed. The physical properties of the intergalactic medium are discussed in the light of recent work on the "Lyman alpha clouds". The uses of the lines for studies of the evolution of clustering in the Universe are described. Recent puzzling results on common absorption in pairs of QSOs, particularly Q1037-2704 and Q1038-2712, are summarized.
\end{abstract}

\section{INTRODUCTION}

It is not possible to talk both about the general properties of absorption lines in QSO spectra and their applications in Cosmology in the allotted time. Therefore, I shall only briefly review what is known about the absorption lines themselves and then concentrate most of my talk on cosmological questions. As it is, some parts of this written contribution were omitted from the talk due to time constraints.

It is currently believed that the quasar absorption lines can be divided into three types, each having a different origin:

1. The "trough" (or BAL) systems. A small proportion of QSOs (perhaps 5\%) have very broad absorption troughs on the blue side of the corresponding emission lines. Lines of very high states of ionization-C IV, Si IV, N V, O VI-are found, in addition to Ly $\alpha$ absorption. The troughs have widths of up to $0.1 \mathrm{c}$. and extend to equivalent velocities of up to $0.2 \mathrm{c}$ from redshift of the QSO.

2. The heavy element systems. These contain sharp lines due to $\mathrm{H}$ and to heavier elements which can be shown to arise in a tenuous gas of near solar composition. Interpreted in terms of relative velocity, the observed values of $z_{e m}-z_{a b s}$ yield velocities ranging from infall to the quasar at a few thousand $\mathrm{km} \mathrm{s}^{-1}$ to outflow speeds of $0.8 \mathrm{c}$.

3. The Ly $\alpha$ systems. All quasars in whose spectra Ly $\alpha$ emission can be observed show an increased density of sharp absorption lines on the blue side of the emission line as compared with that on the red side. Following the original suggestion of Lynds (1971) it is now established that most of the lines on the blue side of Ly $\alpha$ are single Ly $\alpha$ absorption lines; the stronger Ly $\alpha$ lines are accompanied by Ly $\beta$ and sometimes by Ly $\gamma$, but no lines of heavier elements have been observed.

Three additional types of absorption system are sometimes referred to. First there are the "Lyman limit" systems-in which the optical depth at the Lyman discontinuity is greater than or equal to unity. The H I column density is at least $10^{17} \mathrm{~cm}^{-2}$. These 
systems have the advantage that they are easily identified in relatively poor spectra: they appear to be "heavy element" or, rarely, "Ly $\alpha$ forest" systems at the extreme of the distribution of column densities (Tytler 1982; Snijders and Tytler 1987; Sargent and Boksenberg 1983). Secondly, there are the "damped Ly $\alpha$ " systems which have very strong Ly $\alpha$ absorption lines whose profiles imply H I column densities of $10^{20}$ to $10^{22} \mathrm{~cm}^{-2}$. These systems always have associated heavy element lines of primarily lowionization species and are thought to arise in galactic disks (Carswell et al. 1975; Wolfe et al. 1986). Lastly, there appears to be a tendency in some QSOs for clumps of heavy element redshifts of relatively high ionization to occur close to (including, in some cases slightly above) the emission redshift. There is a suspicion that this phenomenon may be associated with radio QSOs (Foltz et al. 1986).

It is generally believed that the "trough" systems represent material ejected from the quasar. (for a review see Weymann and Foltz [1983].) On the other hand, the nature of the "heavy element" and the "Ly $\alpha$ " systems has been more controversial. However, it now seems generally accepted that the majority of the "heavy element" absorption systems are produced by intervening galaxies and that the "Ly $\alpha$ " systems are probably produced by primordial, intergalactic clouds. (For recent reviews see Boksenberg and Sargent [1983] and Sargent and Boksenberg [1983].) It is possible that some other heavy element redshift which appear to clump near the emission redshift are not due to intervening material (Foltz et al. 1986); further studies of their apparent association with radio QSOs will settle this question.

A variety of arguments have shown that the majority of the sharp absorption lines in QSO spectra are produced by cosmologically distributed, intervening objects. Among these we may cite:

1. The Poissonian distribution of the number of absorption redshifts per object in a given redshift range. This was established for the Ly $\alpha$ clouds by Sargent and Boksenberg (1983) and for the C IV systems by Young, Sargent, and Boksenberg (1982).

2. The existence of common absorption systems in QSOs separated by minutes of arc (Shaver and Robertson 1983a).

3. The upper limits on the population of excited fine structure states in ions such as C II and Si II gives limits on the electron density of the absorbing gas which, when combined with estimates of the degree of ionization of the gas, leads to a lower limit on its proximity to the QSO in which the absorption is observed. Typically such distances come out to be $\geq 100 \mathrm{kpc}$, thereby leading to unrealistic energy and momentum requirements if it is supposed that the gas has been ejected from the QSO in which the absorption is observed (Goldreich and Sargent 1976; Sargent et al. 1979).

\section{COSMOLOGICAL APPLICATIONS-OVERVIEW}

The uses of QSO absorption lines in cosmology are only just beginning to be realized in many cases. However, it is useful to list the potential applications for absorption line studies:

1. Physical properties of the interstellar gas in galaxies at large redshifts-particularly the composition, spatial extent and the density of the gas and its division into neutral (H I) and ionized (H II) constituents. Eventually such studies should lead to an understanding of the evolution of the gas in galaxies of various morphological types. Progress in this direction should be considerably accelerated once the Hubble Space Telescope enables us to study absorption systems due to nearby galaxies at the same rest wavelengths as are perforce used for the high redshift observations.

2. Rotation curves of the faint outer parts of galaxies by using suitably placed 
background QSOs as targets. Such observations have considerable potential for work on the cosmological missing mass problem.

3. Physical properties of the general intergalactic medium at large redshifts. This can be done either directly through observations of the Gunn-Peterson (1965) effect, or indirectly through observations of the Ly $\alpha$ clouds, particularly if they are pressure confined. (The inception of the Hubble Space Telescope will permit us to obtain important limits on the properties of the intergalactic medium through observations of the Gunn-Peterson effect in He I and He II as well as H I.) clouds.

4. Evolution of the number density of absorbing objects-galaxies and intergalactic

5. Primordial abundances of D and He through studies of the compositions of the Ly $\alpha$ clouds.

6. Evolution of large-scale structure through observations of the correlation function $\xi(r)$ of galaxies and the $\operatorname{Ly} \alpha$ clouds as a function of redshift.

7. Determination of the deceleration parameter $q_{0}$ from number density counts of particular types of absorption lines.

8. Observations of the intergalactic radiation field at large redshift through its effect on the physical site of the galactic halo absorbers and the Ly $\alpha$ clouds. In addition, it is possible to obtain direct limits on the microwave background temperature at high redshifts through studies of the populations of excited fine structure levels in suitable ions (Meyer et al. 1986).

\section{COSMOLOGICAL EVOLUTION}

\subsection{Theory}

Consider absorbing clouds of cross section $\pi r_{0}^{2}$ and number density $\Phi_{0}$ per unit co-moving volume. It can be shown that the number density of absorption systems of a particular type per unit redshift range is given by

$$
\frac{d N(z)}{d z}=N_{0}(1+z)\left(1+2 q_{0} z\right)^{-1 / 2}
$$

if the cosmological constant $\Lambda=0$ (Peterson 1978; Sargent, Young, Boksenberg, and Tytler 1980). Here

$$
N_{0}=\pi r_{0}^{2} \Phi_{0} \frac{c}{H_{0}}
$$

is the local $(z=0)$ value of $N(z)$. Expression (1) clearly holds if there is a constant size distribution for the absorbing objects at all redshifts. If, on the other hand, the absorbers evolve in constant time then the product $\pi R_{0}^{2} \Phi_{0}$ and hence $N_{0}$ may be functions of redshift. For comparison with observations it is convenient to express equation (1) as a power law:

$$
\frac{d N(z)}{d z}=\text { const. }(1+z)^{\gamma}
$$

It is easy to show that

$$
\gamma=\frac{1+q_{0} z-q_{0}}{1+2 q_{0}}
$$

In particular, $\gamma=1$ for $q_{0}=0$ and $\gamma=1 / 2$ if $q_{0}=1 / 2$. For other values of $q_{0}, \gamma$ is a function of $z$. 
Thus, an empirical plot of $\log (\mathrm{dN} / \mathrm{dz})$ versus $\log (1+\mathrm{z})$ may be used to determine $q_{0}$ if one has independent evidence on the behavior of the product $\pi r_{0}^{2} \Phi$ with redshift.

\subsection{Evolution of the Ly $\alpha$ Clouds}

The best data on redshift evolution for QSO absorption lines comes from the Ly $\alpha$ clouds since their density is so high. Following Peterson's (1978) initial work, all recent studies have confirmed that the Ly $\alpha$ clouds exhibit unquestionable cosmological evolution, although there are still small differences in the results obtained by different investigators for identical data sets which are hard to explain (Murdoch et al. 1986). These last authors find

$$
\frac{d N}{d z} \propto(1+z)^{2.17}
$$

a result which is in line with several earlier studies. It will be noted from equation (4) that an exponent $\gamma=2.17$ is too large to be accommodated with any value of $q_{0}$ and that the Ly $\alpha$ clouds, therefore, must evolve in cosmic time in the sense of either being more numerous or having larger effective cross sections at higher redshifts, or both. (The evolution of the density of the "Ly $\alpha$ forest" may easily be seen by inspecting high quality spectra of QSOs with redshifts around $z \sim 2$ and $z \sim 3$, respectively.) Carswell et al. (1982), Murdoch et al. (1986) and Tytler (1987) have recently discussed a puzzling anomaly in which the line density in most individual QSOs does not show a convincing decrease with redshift over the small range $\Delta z=0.19$ that is accessible between the $\operatorname{Ly} \alpha$ and $\operatorname{Ly} \beta$ emission lines. (Below the Ly $\beta$ emission line the Ly $\alpha$ absorptions are "contaminated" by Ly $\beta$ absorptions corresponding to Ly $\alpha$ lines at higher redshifts and so the situation here is harder to judge.) The origin of this "inverse" effect is obscure and probably indicates that our understanding of the physics of the Ly $\alpha$ clouds is still very insecure. However, one possibility to explain it which has not been explored in the existing literature is that QSOs occupy regions of the Universe which are unsuitable for the formation of $\operatorname{Ly} \alpha$ clouds over large volumes-for example, by having too great a pressure in the intergalactic medium.

\subsection{Evolution of Heavy Element Redshift Systems}

The heavy element redshift systems have a much lower density than the Ly $\alpha$ cloud density and so their evolution is much more difficult to study. Marginal evidence for and against evolution of the C IV redshift systems which can be observed from the ground in the redshift range $1.2 \leq z \leq 4.0$ has been discussed by Bergeron and Boissé (1983) and by Boissé and Bergeron (1985). Tytler (1986) has made an extensive study of Mg II absorption systems in the redshift range $0<z<2$. My interpretation of this data is that there is probably evolution if $q_{0} \sim 1 / 2$ but that if $q_{0} \sim 0$ there is no evidence for evolution. A large new homogeneous survey of C IV systems in the redshift range 1.5 $<z<3.5$ which has recently been completed by Boksenberg and the author should give valuable information on the evolution of the heavy element redshift systems. In any case, it is almost certain from existing data that the density of the Ly $\alpha$ clouds increases more rapidly with redshift than that of the heavy element systems.

\subsection{Evolution of the Lyman Limit Systems}

A particularly interesting class of QSO absorption systems is those which show a measurable discontinuity of the Lyman limit, implying that their H I column density is $N(H I) \geq 10^{17} \mathrm{~cm}^{-2}$. Such systems are almost invariably associated with lines of heavier 
elements at the same redshift. Consequently, it is supposed that they represent the high column density "tail" of the distribution of heavy element redshift systems that are associated with galaxies. One Lyman limit system, at $z_{a b s}=2.99$ in the QSO Pks 2126$158\left(z_{e m}=3.28\right)$, has been found with no observable heavy element lines, despite its high $\mathrm{H}$ I column density; this system is particularly important in attempts to set limits on the heavy element composition of the Ly $\alpha$ clouds (Sargent 1982; Sargent and Boksenberg 1983; Chaffee et al. 1985). The Lyman limit systems have the advantage that they can be detected on low-resolution spectra of relatively low $\mathrm{S} / \mathrm{N}$ ratio and hence it has been possible to study their density down to redshifts as low as $z=0.3$ using the IUE satellite. (Tytler 1982; Snijders and Tytler 1986; Bechtold et al. 1984). Snijders and Tytler (1986) have assembled a list of 40 Lyman limit systems covering the redshift range $0.3<z<3$ in 120 QSOs. The statistical study of these systems is complicated by the fact that the presence of a Lyman limit system at same redshift $z_{1}$, blots out the QSO continuum at all wavelengths below $912\left(1+z_{1}\right) \AA$ so that only one such system can be discovered per QSO. Tytler (1982) has ingeniously modified statistical techniques called "survival statistics", developed by the Insurance industry for a different but analogous problem, to deal with this complication. The density of Lyman limit systems shows a definite increase with redshift which, however, is incompatible with $q_{0} \sim 1 / 2$ and no evolution in the intrinsic physical properties of the absorbers. The data are compatible with $q_{0} \sim 0$. It is interesting and perhaps significant that the density increases with redshift of both the Lyman limit and Mg II absorption systems are compatible with $q_{0} \sim 0$ and no evolution in the product $\pi r_{0}^{2} \Phi_{0}$ with redshift. If $q_{0} \sim 1 / 2$, as the "inflationary" cosmology demands, then the product $\pi r_{0}^{2} \Phi_{0}$ for the Lyman-limit absorbers must increase with $z$ in such a manner as to mimic a $q_{0} \sim 0$ Universe. On the other hand, direct determinations of $q_{0}$ from the density of luminous matter already point to a low-density Universe.

Snijders and Tytler (1986) combined measurements of the Lyman discontinuity and the equivalent widths of the associated Ly $\alpha$ absorption lines in order to determine H I column densities for their sample of 40 Lyman-limit redshift systems. The values are in the range $17<\log N(H I)<21.5$; the distribution $\log \mathrm{N}(\mathrm{H} \mathrm{I})$ appears to be constant over the observed redshift range $0.3<z_{L L S}<3.5$. Thus, there is no evidence from this independent datum that the physical properties of the absorbers change systematically with redshift.

Tytler's work shows that $50 \%$ of randomly chosen lines of sight intercept a cloud which absorbs $99 \%$ or more of the flux at the Lyman limit at a redshift of $z=2$; the number rises to just over $90 \%$ by a redshift of $z=4$. The corresponding numbers for systems which absorb $10 \%$ or more of the flux is $70 \%$ at a redshift of $z=1$ and $100 \%$ at $z=2$. Thus, such systems have a significant effect on the metagalactic flux from radiation capable of ionizing H I. Tytler estimates that the metagalactic ionizing flux from QSOs and galaxies is cut down by a factor of about 3 over the redshift range $2<z<4$ due to this effect.

\section{ABSORPTION AND INTERGALACTIC GAS}

\subsection{The Gunn-Peterson Test}

It was shown by Gunn and Peterson (1965) that the overlapping Ly $\alpha$ absorption lines from any generally distributed intergalactic neutral hydrogen would produce a depression in the level of the QSO continuum on the blue side of the Ly $\alpha$ emission line in a QSO as compared with the level on the red side. The optical depth of the depression is given in 
terms of the H I volume density by

$$
\tau_{\nu}=\frac{8.3 \times 10^{10} n_{H I}(z)}{(1+z)\left(1+2 q_{0} z\right)^{1 / 2}}
$$

Early observations of high redshift QSOs (e.g., $3 \mathrm{C} 9, z_{e m}=2.016$ ) revealed no observable continuum depression. With a conservative estimate $\tau_{\nu}<0.5$ for the optical depth, remarkably low limits could be placed on any intergalactic $\mathrm{H}$ I. Thus, with $q_{0}=$ $1 / 2, n_{H} \leq 3 \times 10^{-11} \mathrm{~cm}^{-3}$ at $z=2$.

Later, Davidson, Hartig, and Fastie (1977) applied the method to space-based observations of 3 C $273\left(z_{e m}=0.158\right)$. They found no evidence for a continuum depression (and no evidence for absorption lines) and were able to obtain a limit on the H I density at the present epoch of $n_{H} \leq 6 \times 10^{-12} \mathrm{~cm}^{-3}$.

The recognition of the existence of the "Ly $\alpha$ forest" in the early 1970 s diverted attention away from the Gunn-Peterson effect because the presence of a rich line spectrum in the blue side of the Ly $\alpha$ emission line makes the determination of the true continuum level more difficult. Recently, however, Steidel and Sargent (1987) have accurately measured the continua of several QSOs with redshifts around $z \sim 3$ and have shown that within the errors the lines do in fact account for all the apparent continuum depression seen on the blue side of Ly $\alpha$ in such objects. It is possible to infer from their data that, conservatively, $\tau \leq 0.05$ at $z=3$. This leads to limits on the density of any generally distributed H I gas of $n_{H} \leq 2.4 \times 10^{-12} \mathrm{~cm}^{-3}$ at $z=3$ if $q_{0}=0$. Dividing by $(1+z)^{3}$ the corresponding limit at $\bar{z}=0$ would be $n_{H}<3.8 \times 10^{-14} \mathrm{~cm}^{-3}$. For $q_{0}=0$ the corresponding limits are twice these values.

\subsection{The Ly $\alpha$ Clouds and the Intergalactic Medium}

The Ly $\alpha$ clouds are thought to be tenuous objects of galactic dimensions which are highly ionized by the intergalactic ionizing flux due to QSOs and galaxies. Sargent, Young, Boksenberg, and Tytler (1980) suggested that they are not self-gravitating but are confined by the pressure of a general intergalactic medium. They pointed out that the clouds could only be self-gravitating if they were very large, so large in fact that they would fill a substantial fraction of the Universe. Sargent et al. studied the possible physical properties of the clouds and the hypothetical surrounding medium using such conditions as the requirement that the hotter and more tenuous medium should not evaporate away the clouds during the Hubble time appropriate to the epoch at which the clouds are observed. Their resulting estimates (all at $z=2.44$ ) were:

For the Ly $\alpha$ clouds: density $n_{H}^{c}=10^{-4} \mathrm{~cm}^{-3}$, temperature $T_{c}=3 \times 10^{4} \mathrm{~K}$, ionization fraction $n_{H I I}^{c} / n_{H I}^{c}=10^{5}$, cloud diameter $D=3 \times 10^{22} \mathrm{~cm}(10 \mathrm{kpc})$, cloud mass $M^{c}=10^{41} \mathrm{~g}=3 \times 10^{7} M_{\odot}$ and space density $\Phi^{c}=2 \times 10^{2} \mathrm{Mpc}^{-3}$. The contribution of the clouds to the cosmological density is small, namely $\Omega^{c}=2 \times 10^{-3}$. $10^{5} \mathrm{~K}$.

For the intergalactic medium: density $n_{H}^{M}=10^{-5} \mathrm{~cm}^{-3}$, temperature $T_{M}=3 \times$

It was pointed out that those parameters are very tight-the clouds would evaporate if more tenuous than the quoted value and collapse if less tenuous. More sophisticated considerations along similar lines by Ostriker and Ikeuchi (1983) and by Black (1981) led to similar results. Later, Ikeuchi and Ostriker (1986) carried out calculations of the evolution of the clouds and surrounding medium in cosmic time which accounted in a satisfactory way for the properties observed at $z \sim 2.5$.

In a beautiful and difficult observation, Foltz, Weymann, Roser, and Chaffee (1984) obtained spectra of the Ly $\alpha$ absorption lines in the two components of the double 
QSO Q2345+007 ( $\left.z_{e m} \sim 2.1\right)$ which are separated by $7^{\prime \prime}$ and which are believed to be the result of gravitational lensing by a so far undetected galaxy or cluster. Foltz et al. showed that, most of the stronger Ly $\alpha$ lines are common to the two components but that a few would appear not to be. This led them to infer a size of about $8 \mathrm{kpc}$ for the Ly $\alpha$ cloudsthe exact value depends on the unknown distance of the lensing galaxy which is assumed to have a redshift of $z \sim 1$. This diameter estimate is seen to be very close to that arrived at by purely astrophysical considerations and would at first sight appear to confirm the picture of pressure confinement for the clouds. However, Rees (1986) proposed that the Ly $\alpha$ clouds are baryonic condensations in "mini-halos" of cold dark matter which serves to confine them gravitationally. This idea is attractive because such mini-halos are the first objects to form in the currently popular cool dark matter dominated cosmologies.

The general intergalactic medium inferred from the pressure confinement arguments would have a temperature $T^{M}=10^{3} \mathrm{~K}$ and a density $n^{M} \sim 2 \times 10^{-7} \mathrm{~cm}^{-3}$ at the present epoch $(z=0)$; it would remain highly ionized as the Universe expands despite its low temperature because the recombination time is longer than the Hubble time. Its contribution to the cosmological density parameter, $\Omega^{M} \sim 0.1$ for $H_{0}=50 \mathrm{~km} \mathrm{~s}^{-1} \mathrm{Mpc}^{-1}$ is somewhat higher than that inferred from visible galaxies and is consistent with the baryonic density $\Omega_{B} \sim 0.14$ required by current primordial nucleosynthesis data (Boesgaard and Steigman 1985). A medium with the quoted parameters is too cool and tenuous to be observed directly by presently conceivable techniques.

While the current situation is not clear, it is certain that the Ly $\alpha$ clouds could not co-exist with a hot, dense intergalactic gas with $T \sim 10^{7} \mathrm{~K}$ and $\Omega^{M} \sim 1$ which is required to account for the diffuse X-ray background.

\section{CLUSTERING OF QSO ABSORPTION LINES}

\subsection{Clustering of Galaxies}

The crudest and most commonly used quantitative measure of the clustering tendency of galaxies is the correlation function $\xi(r)$ introduced by Peebles. The probability $d P$ of finding a galaxy in volume $d V$ at distance $r$ from any given galaxy written in the form

$$
d P=\Phi[1+\xi(r)] d V
$$

where the first term represents the Poissonian expectation from randomly distributed galaxies and $\xi(r)>1$ represents the effect of clustering. Empirically it is found that locally

$$
\xi(r)=\left(\frac{r}{5 \mathrm{Mpc}}\right)^{1.77}
$$

Virtually nothing is known observationally about the evolution of $\xi(r)$ in cosmic time.

It has been clear for some years that in principle we can use QSO absorption lines to study the evolution of the correlation function at large redshifts beyond where we can directly observe typical galaxies. Thus if the absorbers are clustered so should their redshifts be if they are largely due to the expansion of the Universe. (The observed redshifts will, of course, be modified by the peculiar motions induced by the clustering if observations are made along the line of sight to a particular QSO). On the other hand, observations of correlated absorptions in nearby lines of sight leads to complementary information. A little consideration shows that by combining the results of the two types of observation it is possible to determine the distortions in redshift space of which the 
"fingers of God" are one manifestation (Sargent and Turner [1977] have discussed how such characteristic distributions may be used to determine $\Omega$.)

Thus we are led to the idea of looking at the correlation functions in redshift for different types of QSO absorption lines. As a practical matter it is necessary to eliminate the epoch-dependent stretching effects on large scale clustering produced by the expansion of the Universe. Thus, consider two absorbers of redshift $z_{1}$ and $z_{2}$, respectively. We first calculate the proper distance $S_{0}$ of each as measured at the present cosmic epoch for $q_{0}=$ $1 / 2$ :

$$
S_{0}(1 / 2, z)=\frac{2 c}{H_{0}}\left[1-(1+z)^{-1 / 2}\right]
$$

so that for any other epoch $S(z)=S_{0}(z) /(1+z)$. We then calculate the spatial separation

$$
s_{0}=S_{0}\left(z_{2}\right)-S_{0}\left(z_{1}\right) \text {. }
$$

The natural unit of length is the Hubble radius $c / H_{0}=6000 \mathrm{Mpc}$ if $H_{0}=50 \mathrm{~km} \mathrm{~s}^{-1}$ $\mathrm{Mpc}^{-1}$.

\subsection{Clustering of the Heavy Element Redshifts}

The heavy element redshifts are strongly clustered on a scale of $150 \mathrm{~km} \mathrm{~s}^{-1}$. It is not yet clear whether this is due to the relative motion of clouds within particular galaxies (Bahcall and Spitzer 1976) or whether it represents the tip of the galaxian correlation function (Young, Sargent, and Boksenberg 1982). The implications if the latter hypothesis is correct are discussed later in this section. Marginal evidence for clustering of C IV doublets on a scale of $2000 \mathrm{~km} \mathrm{~s}^{-1}$ was found by Young et al. (1982). This, of course, could not be due to motions of discrete clouds within galaxies; one of the most important aims of the large survey by Sargent and Boksenberg which was mentioned earlier to investigate C IV clustering on scales of $300-2000 \mathrm{~km} \mathrm{~s}^{-1}$.

\subsection{Clustering of Ly $\alpha$ Clouds}

Since the Ly $\alpha$ clouds are much more numerous than the objects (presumably galaxies) which produce the heavy element redshifts, it is much easier to investigate their clustering properties. Sargent et al. (1980) showed from a correlation function analysis that there is no observable clustering in the Ly $\alpha$ clouds on all scales from 300 to $30,000 \mathrm{~km} \mathrm{~s}^{-1}$. In a later paper, Sargent, Young, and Schneider (1982) showed that a cross correlation analysis between Ly $\alpha$ absorption lines in the spectra of two QSOs, Q1623+268 and Q1623+267, which are separated by $3^{\prime}$ on the plane of the sky, also show no evidence for clustering in the same range of scales. Sargent et al. (1982) showed that these measurements imply that the "correlation length" for the Ly $\alpha$ clouds in equation (8) is $r_{c} \leq 0.2 \mathrm{Mpc}$ at $z \sim 2.5$. On the "hierarchial" model of the evolution of clustering which has been advocated by Peebles and his associates, and which arises naturally in the "cold dark matter dominated" cosmologies, the correlation function is expected to evolve such that the variation of the correlation length with redshift scales as

$$
r_{c}(z)=r_{c(z=0)}(1+z)^{-5 / 3} \text {. }
$$

Thus, we might expect $r_{c}=1 \mathrm{Mpc}$ at $z=2.5$ on this particalarly simple model. The observed upper limit is a factor of 5 less than this. As we shall see later, one possible explanation is that the Ly $\alpha$ clouds await galaxy clusterings. (At the present Symposium Atwood and colleagues presented evidence that the Ly $\alpha$ clouds cluster weakly on scales 
less than $\Delta v=300 \mathrm{~km} \mathrm{~s}^{-1}$.) This result, if correct, is not in conflict with earlier work. Moreover, it still remains the case that the correlation functions of the Ly $\alpha$ clouds and the heavy element absorbers are remarkably different on scales $\Delta v \sim 150 \mathrm{~km} \mathrm{~s}^{-1}$; this fact alone almost certainly indicates that the two kinds of absorption lines originate in different kinds of object.

(Since this talk was given, a reprint has appeared by Rees and Carswell (1987) drawing attention to the fact that the flat correlation function of the Ly $\alpha$ clouds on scales of several thousand $\mathrm{km} \mathrm{s}^{-1}$ imply that there is no evidence for "voids" in their distribution of the kind which are so prominent in the local distribution of galaxies.)

\subsection{Implications of the Clustering Data}

Salmon and Hogan (1986) have compared the data published by Sargent et al. (1980) regarding the clustering properties of the Ly $\alpha$ clouds and the heavy element absorbers with simulations of the behavior of absorption-line systems in cold dark matter cosmologies. The difference in the clustering tendencies of the two types of absorber can be understood in two possible ways:

1. The heavy element absorption systems are associated with galaxies which in turn constitute an unbiased sample of the overall mass distribution in an $\Omega=0.2$ Universe. On this hypothesis some mechanism must be invented in order to prevent the Ly $\alpha$ clouds also following the mass distribution. A natural possibility, which was pointed to me by J. Ostriker in another connection, is that the intergalactic medium is clustered with the galaxies and that its pressure destroys the clouds. As Ostriker pointed out, the intergalactic pressure in the Coma cluster gas $\left(T \sim 10^{8} \mathrm{~K}, n_{H} \sim 10^{-3}-10^{-4} \mathrm{~cm}^{-3}\right)$ is far in excess of the pressure inferred for the clouds. $\left(n_{H}^{c} T^{c} \sim 1\right.$. Presumably, weaker clusterings have gas with lower values of $n_{H}^{M} T^{M}$.)

2. In an $\Omega=1$ Universe the correlation at $z=3$ is much smaller than that in an $\Omega=0.2$ Universe. The Ly $\alpha$ clouds are then an unbiased sample of the overall mass distribution while the heavy element absorbers, like the galaxies, are more strongly clustered than the mass.

These considerations, inconclusive as they are, point up the enormous potential value of QSO absorption lines in giving information about the clustering of matter at redshifts inaccessible to other modes of observation.

\section{PAIRS OF QSOs AND LARGE SCALE STRUCTURE}

\subsection{The Pair Q0307-199A,B}

The existing studies of common heavy element absorption redshifts in pairs of QSOs offer tantalizing glimpses of large scale organization in the clustering of the absorbers. Shaver and Robertson (1983a) have been particularly active in this kind of investigation. A particularly interesting pair studied by Shaver and Robertson (1983b) is that comprising UM 680 (Q0307-195A; $\left.z_{e m}=2.144\right)$ and UM $681\left(\mathrm{Q} 0308-195 \mathrm{~B} ; z_{e m}=2.122\right)$. These $19^{m}$ objects are separated by $58^{\prime \prime}$ on the plane of the sky; this corresponds to $378\left(H_{0} / 100\right)^{-1}$ kpc at $z=2.13$, assuming $q_{0}=1 / 2$. Shaver and Robertson (1983b) and later Sargent and Boksenberg (1987) have found heavy element absorption redshifts in UM 680 at $z_{a b s}=$ $2.1228,2.0919,2.0353,1.7042$, and 1.5623 , respectively. In UM 681 they found redshifts at $z_{a b s}=2.1220,2.0323,1.7057$, and 1.7885 , respectively. It will be seen that the spectra contain common redshifts at $z_{a b s}=2.12,2.03$, and 1.70 .

Shaver and Robertson $(1983 b)$ pointed out that the common system at $z_{a b s}=$ 
2.12 is likely to be a very large cloud associated with UM 681 . The velocity difference $\Delta v=c \Delta z / 1+z$ between the emission redshift of UM 681 and the mean of the absorption redshifts observed in the two QSOs is $\Delta v$ (em. - abs.) $=29 \pm 29 \mathrm{~km} \mathrm{~s}^{-1}$. If it is supposed that the absorbing gas is in the form of a disk about $1 \mathrm{Mpc}$ in diameter around UM 681 then we may estimate that the total mass of the gas is $2 \times 10^{10} M_{\odot}$. The velocity difference between the absorption systems at $z_{a b s}=2.1228$ in UM 680 and $z_{a b s}=2.1220$ in UM 681 turns out to be $\Delta v(A-B)=77 \pm 14 \mathrm{~km} \mathrm{~s}^{-1}$. If this velocity difference is due to rotation of the hypothetical disk, then its total mass is

$$
M_{t}=\frac{10^{11}-10^{12}}{h \sin ^{2} i} M_{\odot}
$$

where $h=\left(H_{0} / 100\right)$ and $i$ is the angle of inclination of the disk to the line of sight to UM 681. This object is reminiscent of the enormous cloud of ionized gas around the radio source MR 2251-178 which was discovered by Bergeron et al. (1983).

The other two absorption systems, at $z_{a b s}=2.03$ and $z_{a b s}=1.70$, respectively, are clear cases of common absorption which is not associated with either QSO. They could be due to galaxies in intervening clusters or superclusters or to the extended halos of single galaxies. In either case, the velocity differences $\Delta v(A-B)$ of $-297 \mathrm{~km} \mathrm{~s}^{-1}$ (for $z_{a b s}=$ 2.03 ) and $\Delta v=172 \mathrm{~km} \mathrm{~s}^{-1}$ (for $z_{a b s}=1.70$ ) point to the existence of substantial mass on large scales-as is also implied by the flat rotation curves of nearby galaxies and the large velocity differences between nearest neighbor pairs of galaxies in our immediate vicinity (Davis, Huchra, Latham and Tonry 1982).

Crotts (1985) favors the notion that these correlated absorptions are all produced by very extended absorbers. He estimates on the basis of correlation function simulations that these large objects have about the same space density as clusters in the Zwicky catalogue $\left(\sim 10^{-4} \mathrm{Mpc}^{-3}\right)$ and a correlation scale length of about $500 \mathrm{kpc}$ which does not change with redshift over the observed range.

\subsection{Absorption in a QSO in the Field of Pks $0237-233$}

The $16.8 \mathrm{mag}$ QSO Pks 0237-233 $\left(z_{e m}=2.220\right)$ was one of the first to have its absorption spectrum studied in some detail, both because the QSO is abnormally bright and because its absorption spectrum is unusually rich. In particular, it has close double absorption redshifts at $z_{a b s}=1.59,1.61,1.63$ and 1.65 and a prominent "clump" of at least 7 redshifts at $z_{a b s}=1.67$ which extends over a range corresponding to $\Delta v \sim 5000 \mathrm{~km} \mathrm{~s}^{-1}$ in velocity. In 1977, following the completion of the work on the absorption spectrum of Pks 0237-233 by Boroson, Sargent, Boksenberg, and Carswell (1978), Boksenberg and the present author realized that such redshift clumps would correspond to relatively large angles on the sky if they corresponded to large structures such as superclusters of galaxies. For example, at $z=2$ a structure of size $50 \mathrm{Mpc}\left(H_{0}=50 \mathrm{~km} \mathrm{~s}^{-1} \mathrm{Mpc}^{-1}\right)$ subtends an angle of order 1 degree on the plane of the sky if $q_{0}=1 / 2$ and about $0.5 \mathrm{deg}$ if $q_{0}=0$. Accordingly, we embarked on a systematic survey for QSOs in the field around Pks 0237233. Two methods were used. In the first one the field around Pks 0237-233 was photographed through U,B,V filters with the Palomar 48-inch Schmidt telescope on a single plate with the telescope being moved between the three exposures which were arranged so as to give roughly equal images for typical stars. These multiple exposure plates were then searched for abnormally blue objects by C. Kowal; his resulting candidate QSOs were then observed spectroscopically at low resolution by Sargent and Kunth at the Palomar 200-inch and Las Campanas 100-inch telescopes.

In a parallel search, A. Boksenberg examined an objective prism plate of the 
field which had been obtained by the UK Schmidt Unit. His QSO candidates around Pks 0237-233 were also observed by Sargent and Kunth.

The result of this work (which is described in more detail in Sargent, Boksenberg, and Kunth [1987]) is that a 19 mag QSO Q0236-234 with a redshift $z_{\text {em }}=2.180$ was discovered $30^{\prime}$ away from Pks 0237-233. High-resolution spectra of this object, secured by Boksenberg at the Anglo-Australian telescope, revealed a spectrum generally devoid of absorption lines longwards of the $\operatorname{Ly} \alpha$ emission line. However, there is a weak C IV doublet at a redshift $z_{a b s}=1.5953$ which coincides exactly with one component $z_{a b s}=$ 1.5950 of one of the double absorption systems in Pks 0237-233. The separation of the lines of sight to the two QSOs on the plane of the sky at $z=1.6$ is $19 \mathrm{Mpc}$ (assuming $H_{0}$ $=50 \mathrm{~km} \mathrm{~s}^{-1} \mathrm{Mpc}^{-1}$ and $q_{0}=1 / 2$ ) evaluated at the epoch when the light traversed the absorbers. The corresponding linear separation evaluated at the present epoch is $50 \mathrm{Mpc}$. This coincidence again points to the existence of large-scale structures at high redshifts and points up the possibility of mapping them through observations of absorption lines in suitable background QSOs.

\subsection{The Wide Pair Q1037-2704, Q1038-2712}

An intriguing case of several common absorption redshifts in the quasar pair Q1037-2704 and Q1038-2712 has recently been discovered by Jakobsen, Perryman, Ulrich, Macchetto, and di Serego Alighieri (1986). The pair is separated by $17^{\prime}$ on the plane of the sky; this corresponds to $4.3 \mathrm{Mpc}$ (assuming $H_{0}=100 \mathrm{~km} \mathrm{~s}^{-1} \mathrm{Mpc}$ and $q_{0}=0.5$ ) at $z \sim 2$. In March 1986 the writer and C. C. Steidel obtained spectra of resolution $2 \AA$ of the pair at the Las Campanas DuPont reflector (Sargent and Steidel 1987). The following is a brief summary of the combined results, most of the essentials of which were described on the basis of lower dispersion spectra by Jakobsen et al. 2704).

1. The emission redshifts are $z_{e m}=2.331(\mathrm{Q} 1038-2712)$ and $z_{e m}=2.193(\mathrm{Q} 1037-$

2. Both QSOs have exceptionally rich absorption spectra. In the recent highresolution survey by Sargent and Boksenberg (1987) referred to earlier no QSO was found with an absorption spectrum as complex as either Q1037-2704 or Q1038-2712. The absorption redshifts in Q1037-2704 occur at $z_{a b s}=1.9125,1.9718,2.0279,2.0708,2.0825$, 2.1279 , and 2.1390 , respectively. Those in Q1038-2712 occur at $z_{a b s}=1.895,1.9550$, $2.0138,2.065,2.077$, and 2.146 , respectively.

3. All the absorption systems contain sharp lines (including C IV doublets in all cases) except for the $z_{a b s}=2.065,2.077$ complex in the spectrum of Q1038-2712 which is a broad feature with some structure, similar to the broad absorption "troughs" found the "BAL" QSOs.

4. There are six pairings: cases in which similar absorption redshifts occur in both QSOs. The calculated velocity differences $\Delta v=c \Delta z /(1+z)$ between the various pairs of systems range from 1800 to $540 \mathrm{~km} \mathrm{~s}^{-1}$; they do not all have the same sign.

5. Several other QSO candidates were discovered in the field of Q1037-2704 and Q1038-2712 by Bohuski and Weedman (1979). One of these, Q1038-2707, is only $5^{\prime}$ away from Q1038-2712. Sargent and Steidel (1987) obtained low-resolution spectra of this object and found that it has a redshift $z_{e m}=1.937$ and that, even at low resolution, there is a strong absorption system at $z_{a b s}=1.89$.

Jakobsen et al. (1986) discussed three hypotheses which might explain the facts summarized heretofore. The first is that the resemblance in the absorption spectra of the two objects is purely a chance occurrence. As they pointed out this is unlikely because both spectra are abnormally rich; however, this is hard to quantify post hoc.

A second possibility is that the redshifts are due to material which has been 
ejected from one or both QSOs. There are several arguments against this hypothesis. Based on their redshifts, Q1037-2704 is $22 \mathrm{Mpc}$ closer to us than Q1038-2712. Since the absorptions seen in both spectra extend only as high as $z_{a b s}=2.146$, a little less than the emission redshift of Q1037-2704 $\left(z_{e m}=2.193\right)$, it is fairly evident that Q1038-2712 is unlikely to be the source of the hypothetical ejection. Otherwise, it is somewhat curious that no absorption redshifts lying between $z_{a b s}=2.33$ and $z_{a b s}=2.19$ are observed in its spectrum. Moreover, it is easy to show that a shell of typical column density $(N(H)=$ $10^{19} \mathrm{~cm}^{-2}$ ) with a radius greater than $22 \mathrm{Mpc}$ leads to completely unreasonable energy and momentum outflows from Q1038-2712. Accordingly, let us consider the consequences of supposing that the ejection occurred instead from Q1037-2704. In this case the ejected matter must extend more than $4 \mathrm{Mpc}$ from the source in order to cover both lines of sight. However, we do not observe any absorptions with redshifts larger than $z_{a b s}=2.19$ in the spectrum of the more distant QSO, Q1038-2712, which would correspond to ejection in its direction. Thus, we are left with the highly artificial picture that the ejection from Q1038-2712 is all in the direction towards us. Even if we accept this possibility, the ejected blobs corresponding to each of the six redshift systems in common must have an extension of at least $4 \mathrm{Mpc}$ perpendicular to the direction of ejection. It is easy to calculate that a typical system must contain about $10^{11} M_{\odot}$ moving at $3200 \mathrm{~km} \mathrm{~s}^{-1}$ away from the source with a resulting kinetic energy of $\sim 10^{61}$ ergs (Sargent and Steidel 1987). If the ejection has been through radiation pressure then the energy requirements must be even higher, because a single photon can transmit in a single scattering at most a fraction $v / c$ of its energy into kinetic energy. Thus, it turns out that for a typical absorption system the energy required for the corresponding blob to be radiatively driven is $10^{64}$ $10^{65}$ ergs. Thus the QSO would be required to radiate more than $10^{47} \mathrm{ergs} \mathrm{s}^{-1}$ for the age of the Universe!

Jakobsen et al. proposed that the common absorptions might be the result of independent ejections from the two QSOs and that some kind of line-locking might be operating between systems such that only one coincidence in redshift would be required rather than several. However, the improved spectra obtained by Sargent and Steidel (1987) show that the ratios of $(1+z)$ between adjacent systems are not the same in both QSOs. Moreover, the absence of absorption from excited fine structure states in the sharp redshift systems implies that the absorbing clouds must still be $\sim 100 \mathrm{kpc}$ from the two QSOs even if independent ejection has occurred. This again leads to severe energy and momentum problems, although they are not as bad as the ones encountered in the preceding arguments.

The remaining possibility is that the unusual density of absorption lines in the two spectra is due to the fact that the two lines of sight are traversing a large scale distribution of galaxies which extends roughly from $z=2.19$ to $z=1.89$. On this hypothesis Q1037-2704 is close to the far side of this structure, while the third QSO, Q1038-2707 is close to the near side. The object must have an extent of about $50 \mathrm{Mpc}$ along the line of sight and be at least $5 \mathrm{Mpc}$ across in the perpendicular direction. (The quoted dimensions are those appropriate to the epoch when the light traversed the system.) The abnormal richness of the absorption systems in the spectra of Q1037-2704 and Q10382712 also implies that the structure must be rare. The least implausible conclusion is that we are observing a supercluster from a particular rare direction. For example, we could be looking along a filament of galaxies in which clusters are embedded.

Although the above explanation of the absorption spectra of Q1038-2712, Q10372704 , and Q1038-2707 is the most plausible of the possibilities, we are nevertheless left with an uncomfortable feeling that none of the proposed explanations are correct. However, if the large-scale structure explanation is essentially correct, we have the fascinating possibility of determining something about the intricate details of superclusters 
at large redshifts. On the other hand, the interpretation of the statistical behavior of the absorption systems observed along different lines of sight in the Universe will be much more complicated than has hitherto been realized.

\section{EPILOGUE}

It is clear from the considerations outlined in this talk that the full exploitation of QSO absorption lines for cosmological studies of various kinds, particularly the correlation and large-scale structure investigations, requires a large body of statistical data which are only now beginning to be obtained. Nevertheless, the absorption offer bright hopes of leading to important information on the distribution of clouds and galaxies at much earlier epochs.

Acknowledgements: I thank my co-workers A. Boksenberg, D. Kunth and C. Steidel for allowing me to quote the results of our joint work in advance of publication. I also thank D. Tytler for providing me with details of his unpublished work. The work described in this paper was supported in part by the National Science Foundation under Grant AST84-16704.

\section{REFERENCES}

Bechtold, J., Green ,R.F.,Weymann, R.J., Schmidt, M., Estabrook, F.B., Sherman,R.D., Wahlquist, H.D., and Heckman, T.M. 1984, Ap.J., 281, 76.

Bergeron ,J., Boksenberg, A., Dennefeld, M., and Tarenghi, M. 1983, M.N.R.A.S., $202,125$.

Bergeron, J. and Boissé, P. 1983, in Quasars and Gravitational Lenses, Proc. 24th Liège Symposium, p. 589.

Black, J.H. 1981, M.N.R.A.S., 197, 553.

Boesgaard, A.M., and Steigman, G. 1985, Ann. Rev. Astr. Ap., 23, 319.

Bohuski, T.J., and Weedman D.W. 1979, Ap. J., 231, 653.

Boissé, P., and Bergeron, J. 1985, Astr. Ap., 145, 59.

Boksenberg, A., and Sargent, W.L.W. 1983, in Quasars and Gravitational Lenses, Proc. 24th Liège Symposium, p. 500.

Boroson, T., Sargent, W.L.W., Boksenberg, A., and Carswell, R.F. 1977, Ap. $J ., 220,772$.

Carswell, R.F, Hilliard, R.L., Strittmatter, P.A., Taylor, D.J., and Weymann, R. 1975, Ap. J., 196, 351.

Carswell, R.F., Whelan, J.A.J., Smith, M.G., Boksenberg, A., and Tytler, D. 1982, M.N.R.A.S., 198, 91.

Chaffee, F.H, Foltz, C.B., Roser, H.-J., Weymann, R.J., and Latham, D.W. 1985, Ap. J., 292, 362.

Crotts, A.P. 1985, Ap. J., 298, 732.

Davidsen, A. , Hartig, G.F. and Fastie, W.G. 1977, Nature, 269, 203.

Davis, M., Huchra, J. Latham, D.W., and Tonry J. 1982, Ap.J., 253, 423.

Foltz, C.B., Weymann, R.J., Peterson, B.M., Sun, L., Malkan, M.A., and Chaffee, F.H. 1986, Ap. J., 307, 504.

Foltz, C.B., Weymann R.J., Roser, H.-J., and Chaffee, F.H. 1984, Ap. J. (Letters), 281, L1. 
Goldreich, P., and Sargent, W.L.W. 1976, Comments on Ap., 6, 133.

Gunn, J. E., and Peterson, B.A. 1965, Ap. J., 142, 1633.

Ikeuchi, S., and Ostriker, J.P. 1986, Ap. J., 301, 522.

Jakobsen, P., Perryman, M.A.C., Ulrich, M.H., Macchetto, F., and di Serego Alighieri, S. 1986, Ap. J. (Letters), 303, L27.

Lynds, C.R. 1971, Ap. J. (Letters), 164, L73.

Meyer, D.M., Black, J.H., Chaffee, F.H., Foltz, C.B., and York, D.G. 1986, Ap. $J$. (Letters), 308, L37.

Murdoch, H.S., Hunstead, R.W., Pettini, M., and Blades, J.C. 1986, Ap. J., 309, 19.

Ostriker, J.P., and Ikeuchi, S. 1983, Ap. J. (Letters), 268, L63.

Peterson, B.A. 1978, in IAU Symposium No.79, Large Scale Structure of the Universe, eds. M.S. Longair and J. Einasto, (Dordrecht: Reidel), p. 390.

Rees, M.J. 1986, M.N.R.A.S., 218, 25P.

Rees, M.J. and Carswell, R.F. 1987, M.N.R.A.S., in press.

Salmon, J., and Hogan, C. 1986 Ap. J., in press.

Sargent, W.L.W. 1982, Phil. Trans Roy.Soc. London A, 307, 87.

Sargent, W.L.W., and Steidel, C.C. 1987, Ap. J., in preparation.

Sargent, W.L.W., and Turner, E.L. 1977, Ap. J. (Letters), 212, L3.

Sargent,W. L. W., Boksenberg, A. and Kunth, D. 1987, Ap. J., in preparation.

Sargent, W.L.W., and Boksenberg, A. 1983, in Quasars and Gravitational Lenses, Proc. 24th Liège Symposium, p. 518.

Sargent, W.L.W., and Boksenberg, A. 1987, Ap. J., in preparation.

Sargent, W.L.W., Young, P.J., Boksenberg, A., and Tytler, D. 1980, Ap. J. Suppl., 42, 41.

Sargent, W.L.W., Young, P.J., and Schneider, D.P. 1982, Ap. J., 256, 374.

Sargent, W.L.W., Young, P.J., Boksenberg, A., Carswell, R.F., and Whelan, J.A.J. 1979, $A p$. J., $\mathbf{2 3 0 , 4 9 .}$

Shaver,P.A., and Robertson, J.G. 1983a, in Quasars and Gravitational Lenses, Proc. 24th Liège Symposium, p. 598.

Shaver, P.A., and Robertson, J.G. 1983b, Ap. J. (Letters), 268, L57.

Snijders, A. and Tytler, D. 1987, in preparation.

Steidel, C.C., and Sargent, W.L.W. 1987, Ap. J., in press.

Tytler, D. 1982, Nature, 298, 427.

Tytler, D. 1987, in preparation.

Weymann, R.J., and Foltz, C.B. 1983 in Quasars and Gravitational Lenses, Proc. 24th Liège Symposium, p. 538.

Wolfe, A.M., Turnshek, D.A., Smith, H.E., Cohen, R.D. 1986, Ap. J. Suppl., $61,249$.

Young, P.J., Sargent, W.L.W., and Boksenberg, A. 1982, Ap.J. Suppl., 48, 445. 
DISCUSSION

BAHCALL: The large scale structure and motion suggested by the absorption lines of the close QSOs is similar to that we find in rich superclusters, with typical scale of $\sim 25 \mathrm{~h}^{-1} \mathrm{Mpc}$ and velocity dispersion of $\sim 1000-2000 \mathrm{~km} / \mathrm{s}$. Since such systems have a reasonably small volume density, are they consistent with the QSO statistics of only occasionally finding such signatu res in QSO spectra.

SARGENT: I think that the structure deduced from the observations of Q1037-2704 and Q1038-2712 is an exceptional and rare one.

SILK: Can you comment on the heavy element abundances and on the ionization state in the six multiple absorption-line systems common to the quasar pair with 17 arc-min separation?

SARGENT: No detailed studies have been carried out yet; however, based on my experience with similar absorption systems in other QSOs I would say that the heavy element abundances will came out solar or only slightly below.

RUBIN: What can you say concerning rotation curves at large $r$ ?

SARGENT: Some years ago Arp discovered a 16 magn. QSO, Q0958+68Wl, about a degree SE of the center of M8I and on the edge of the outer contours of the 21 cn map done at Jodrell Bank. Observations by Boksenberg and I show clear evidence of CaII $\mathrm{H}$ and $\mathrm{K}$ absorption in the spectrum of the QSO at zero redshift and at about $-130 \mathrm{~km} \mathrm{~s}^{-1}$. The latter is due to M81 and shows that a flat rotation curve continues out to at least 4 Holmberg radii.

DEKEL: Why don't we see large lumps like the last case you've shown us in the general correlation function of L $\alpha$ clouds.

SARGENT: It appears that the Lyman $\alpha$ clouds are distributed differently to galaxies. One possibility is that they occupy the "voids" in the galaxy distribution.

PFCKER: I was (last year!) rather convinced by the paper by Leona Marshall-Libby et al., in which the Ly $\alpha$ forest was shown to be of an intrinsic origin. Now, it seems that the $z$-dependence of the forest has forced authors to accept the ided of intervening absorbers. The 
exponent $\gamma$ of the laws $\mathrm{dN} / \mathrm{dz}$ has then been used for some cosmological discussion. But is it not true that the two views are not contradictory but complementary, that there are two populations of absorption lines, and, as a consequence, that we should be very careful about using the coefficient $\gamma$ in cosmological discussions.

SARGENT: Dr。 Libby's work was shown to be unsound by Zuiderwijk (A。J。89, 1808, 1984)。 I believe that the evidence strongly suggests that both the Lyman $\alpha$ forest and the heavy element lines have an intervening origin. 